\title{
The Origin of Volatility Cascade of the Financial Market
}

\author{
Chunxia Yang $^{1}$, Yingchao Zhang ${ }^{1}$, Hongfa $\mathrm{Wu}^{1}$, and Peiling Zhou ${ }^{2}$ \\ ${ }^{1}$ School of Information and Control Engineering, \\ Nanjing University of Information Science and Technology, Nanjing Jiangsu, 210044, \\ P.R. China \\ ${ }^{2}$ Department of Electronic Science and Technology, \\ University of Science and Technology of China, Hefei Anhui, 230026, P.R. China
}

\begin{abstract}
Based on the self-organized dynamical evolutionary of the investors structure, a refined dissipation market model is constructed. Unlike multifractal cascade-like ideas, this model provides a realistic (agent based) description of financial markets and reproduces the same multifractal scaling properties of price changes as the real, which indicate that the self-organized dynamical evolutionary of the investors structure may be the origin of the volatility statistical structure.
\end{abstract}

Keywords: self-organization, multifractal, cascade, financial market model, volatility.

\section{Introduction}

The modelling of random fluctuation of asset prices is of obvious importance in finance, which can be used in practice for volatility filtering and option pricing. The universal features of price changes have attracted widespread attention and concern to construct a useful model. The simplest feature of financial time series, uncovered by Bachelier in 1900, is the linear growth of the variance of the return fluctuations with time scale[1]. More precisely, if one denotes $V(t)$ the price of an asset at time $t$, the return $r_{\tau}(t)$ at time $t$ and scale $\tau$ is simply the relative variation of the price from $t$ to $t+\tau: r_{\tau}(t)=(V(t+\tau)-V(t)) / V(t) \simeq \ln V(t+$ $\tau)-\ln V(t)$; if $m \tau$ is the mean return at scale $\tau$, the following property holds to a good approximation:

$$
\left\langle\left(r_{\tau}(t)-m \tau\right)^{2}\right\rangle_{e} \simeq \sigma^{2} \tau
$$

where \langle\rangle$_{e}$ denotes the empirical average. This behavior typically holds for $\tau$ from a few minutes to a few years, equivalent to the statement that relative price changes are uncorrelated. Linear growth of the variance of the fluctuations with time is typical of the Brownian motion which was proposed as a model of market fluctuation by Bachelier. In this model, returns are not only uncorrelated but actually independent and identical Gaussian random variables. However, due to intensive statistical studies during the last decade, this model suffers the impugnation and the challenge of actual financial data such as the real-life markets are 
of return distributions displaying peak-center and fat-tail properties [2 34], one can observe volatility clustering and a non-trivial "multifractal" scaling [5 6/7], and so on. These universal features portray a world of non Gaussian random walks that Mandelbrot started exploring for us in the sixties, charting out its scrubby paths, on which increasing scientists devote themselves to look for the origin of the market fluctuation 68 891011/2]. One of the contributions is that Mandelbrot's cascades have been used to account for scale invariance properties in statistical finance 9]. Recently, Bacry, Muzy and Delour introduced a modified model that captures the essence of cascades (BMD model) [12. All these models explains the multi-scaling property through the notion of cascade from coarse to fine scales. But, such description using multifractal, cascades-like ideas is still mostly phenomenological. Here, without any postulate, we proposed a model with the same multifractal scaling properties as the reality which arise from a realistic (agent based) description of financial markets and help understand the underlying mechanisms.

\section{$2 \quad$ Model}

Cont and Bouchaud successfully applied percolation theory to modeling the financial market (CB model), which is one of the simplest models able to account for the main stylized fact of financial markets, e.g. fat tails of the histogram of log-returns 13. Based on it, our model incorporates the following components different from the original CB model: (1) A cluster, defined as a set of interconnected investors, grows in a self-organized process. (2) The effect of "herd behavior" on the trade-volume is magnified step by step during the cluster's self-organized accumulating process. (3) Some encountering smaller clusters will form a bigger cluster through cooperating or one defeating the rivals. (4) An infinite cluster may exist without the need to tune $p$ to $p_{c}$ and its trade activity influences price fluctuation. (5) The number of investors participating in trading will vary dynamically.

\subsection{Dynamic of Investor Groups}

Initially, $M=100$ investors randomly take up the sites of a $L * L$ lattice. Then for each cluster, a strategy is given: buying, selling, or sleeping, which are denoted by $1,-1$ and 0 respectively. In reality, the circle of professionals and colleagues to whom a trader is typically connected evolves as a function of time: in some cases, traders are following strong herding behavior and the effective connectivity parameter $p$ is high; in other cases, investors are more individualistic and smaller $p$ seems more reasonable. In order to take the complex dynamics of interactions between traders into account, we assume that it undergoes the following evolution repeatedly:

(1) Growth: most of investors would like to imitate the strategies which have been adopted by many others, which induces "herd behavior" occurring. In this sense the herd behavior is amplified. Specially, the affection of the herd behavior 
will be magnified gradually with the increase of the number of investors adopting this strategy, i.e., with the growth of the clusters. During cluster's growth, a number of new investors will be attracted by it and become its members. In other words, every cluster will absorb new investors with the probability:

$$
P_{d}(\tau)=P_{d}(\tau-1)+k\left(N_{T}-N(\tau-1)\right)
$$

where $k$ is a kinetic coefficient controlling the growth speed and $N_{T}$ is a threshold parameter (It has been validated that the value of the parameters $k$ and $N_{T}$ could be any value. These two parameters have no effects on the self-organization process of the clusters [14] $). N(\tau-1)$ is the number of the agents along it's boundary, defined as a set made up of agents which belong to a cluster and at least border on a site which isn't part of this cluster, at the last time step $\tau-1$. The new participating investor will take up the empty sites around the old clusters and imitate the same strategy as that of it. The probability $P_{d}$ is obviously limited to the range $[0,1]$ so that we have to impose $P_{d}=0$ and $P_{d}=1$ if the recurrence relationship Equ (2) gives values for $P_{d}<0$ or $P_{d}>1$.

(2) New cluster's birth: some investors will randomly and independently enter the market with the probability $P_{n}$. These investors don't belong to an arbitrary existing cluster and will take up the empty sites.

(3) Cooperation: encountering clusters will operate cooperation and confliction between them. When their strategies are same, they are thought to cluster together to form a new group of influence. Or there would be confliction between them. The consequence of confliction is that losers would be annexed by the winner and that a new and bigger cluster whose strategy inherent the winner's will be formed. The probability of cooperation or confliction is as follow, i.e., some a cluster will cooperate with or defeat others with the probability

$$
P_{m}(k)=\frac{\left|s_{\tau}^{k}\right|}{\sum_{j=1}^{n}\left|s_{\tau}^{j}\right|} .
$$

where $\left|s_{\tau}^{j}\right|$ is the size of $\mathrm{j}$-th cluster at time step $\tau$.

(4) Metabolism: in reality, no matter how huge has the size of a group ever been it would collapse due to different influences such as government decision on war and peace. Some new clusters will come into the world in the wake of aging clusters' death. The probability with which an aging cluster will die is:

$$
P_{o}=\frac{x+y}{2 L}
$$

where $x$ or $y$ is the width of this cluster occurring on the lattice in the $x$ or $y$ direction. Equ.(4) indicates that the probability with which a cluster disbands would increase with the cluster growth. Once a spanning cluster exists, it would surely die. When a cluster disbands, all its members would leave the market and the sites where the death cluster ever occupied will be taken up by new investors 
with the probability $P_{n}$. Such occupied sites form a few new clusters. Every new cluster would be given a strategy randomly.

Although each cluster could trade with others at every trading step, the evolutionary frequency of the network topology should not be so often. Thus, we assume that the network structure of the market composed by investor groups would evolve every $N$ trading steps. With the evolutionary of this artificial stock market, the number of investors participating in trading isn't constant. The network will take on different structure; the affection of the herd behavior on the trade-volume is gradually magnified. Cooperation and confliction among clusters are always operating. Without any artificial adjustment of the connectivity probability $p$ to $p_{c}$, spanning cluster may exist, whose activity would influence the price fluctuation.

\subsection{Trading Rules}

Each cluster trades with probability a (called activity); if it trades, it gives equal probability to a buying or selling with demand proportional to the cluster size. The excess demand is then the difference between the sum of all buying orders and selling orders received within one time interval. The price changes from one time step to the next by an amount proportional to the excess demand. To explain the "volatility", Stauffer introduces the feedback mechanism between the difference of the "supply and demand" and activity of the investors [15]. Whereas in our model, the difference of the "supply and demand" not only affects the activity probability but also the probability with which the active clusters choose to buy or sell. The probability $a$ evolves following

$$
a(t)=a(t-1)+\operatorname{lr}(t-1)+\alpha .
$$

where $r$ is the difference between the demand and supply, $l$ denotes the sensitivity of $a$ to $r$ and $\alpha$ measures the degree of impact of external information on the activity. $\alpha \in[-0.005,0.005]$ is a random variable obeying Gaussian distribution of mean value 0 and standard deviation 1. Each active cluster choose to buy or sell with probabilities $2 a(t)\left(1-p_{s}(t)\right)$ and $2 a(t) p_{s}(t)$ respectively. For $r>0$, $p_{s}(t)=0.5+d_{1} r(t-1)$, while for $r<0, p_{s}(t)=0.5+d_{2} r(t-1)$. According to Kahneman and Tversky, it is asymmetry that agents make their decisions when they are in face of gain and loss [16]. When $|r|$ varies within certain range, the degree of depression following some lost is twice that of happiness following the same quantity of profit. Therefore in our model we assume $d_{2}=2 d_{1}$. The difference between the demand and supply is:

$$
r(t)=\sum_{j=1}^{m} \operatorname{sign}\left(s_{t}^{j}\right)\left(\left|s_{t}^{j}\right|\right)^{\gamma} .
$$

where $m$ is the total number of clusters occurring on the market and $\left|s_{t}^{j}\right|$ is the size of $\mathrm{j}$-th cluster at trading time step $t . \gamma$ measures the degree of impact of each cluster's trade-volume on the price, $0<\gamma<1$ allowing for a nonlinear 
dependence of the change of (the logarithm of the) price as a function of the difference between supply and demand 17]. So the evolution of the price is:

$$
V(t)=V(t-1) \exp (\lambda r(t)) .
$$

where $\lambda$ is a coefficient adjusting the market fluidity.

\section{Simulation and Analysis}

Here we set $a(0)=0.09, r(0)=0, V(0)=1, P_{d}(0)=0.4, k=0.0001, N_{T}=50$, $l=\lambda=\frac{1}{L^{2}}, L=100, d_{1}=0.00005, \gamma=0.8, P_{n}=0.6, M=100, N=50$. The model can iterate for a period of any length. More simulations have been done indicating that the return distribution of the present model obeys Lévy form in the center and displays fat-tail property, in accord with the stylized facts observed in real-life financial time series. Furthermore, this model reveals the power-law relationship between the peak value of the probability distribution and the time scales in agreement with the empirical studies on the Hang Seng Index 18. For the Lévy stable processes first suggested by Mandelbrot as an alternative, the return distribution is identical (up to a rescaling) for all $\tau$. As mentioned in empirical studies, the distribution of real returns is not scale invariant, but rather exhibits multi-scaling, in the sense that higher moments of price changes scale anomalously with time:

$$
M_{q}(\tau)=\left\langle\left(\left|r_{\tau}(t)-m * \tau\right|\right)^{q}\right\rangle_{e} \simeq A_{q} \tau^{\zeta_{q}} .
$$

where the index $\zeta_{q}$ is not equal to the Brownian walk value $q / 2$ [8/901011 12 . References [19] illustrates the empirical multifractal analysis of Standard and Poor's Gomposite Index return. The estimated spectrum $\zeta_{q}$ has a concave shape that is well fitted by the parabola:

$$
\zeta_{q}=q\left(1 / 2+\lambda^{2}\right)-\lambda^{2} q^{2} / 2 .
$$

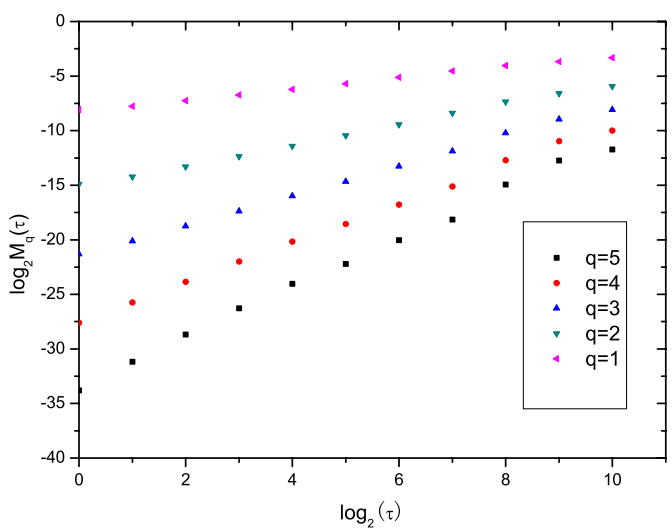

Fig. 1. First five absolute moments of the model as a function of the time period $\tau$ in double logarithmic representation 


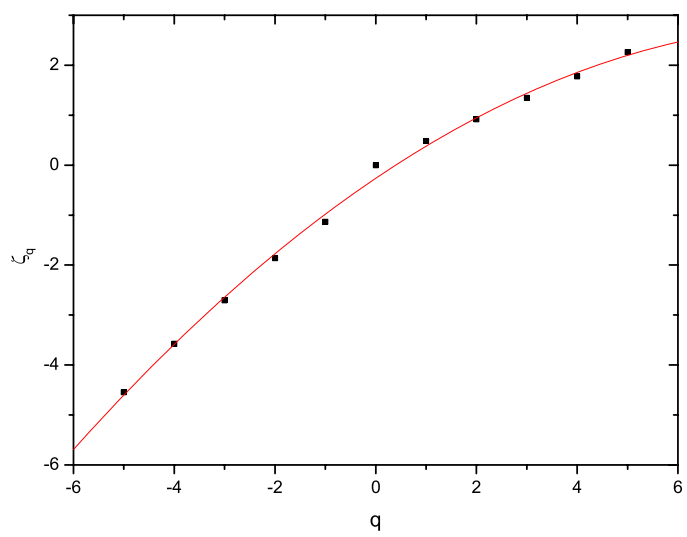

Fig. 2. $\zeta_{q}$ spectrum estimate versus $q$

where $\lambda^{2} \simeq 0.03$. The coefficient $\lambda^{2}$ that quantifies the curvature of the $\zeta_{q}$ is called, in the framework of turbulence theory, the intermittency coefficient. Correspondingly in the finance parlance, $\lambda^{2}$ measures the intensity of volatility fluctuations.

The multifractal scaling properties of our model are numerically checked in Fig. 1 and Fig. 2 where one can recover the same features as that of the Standard and Poor's Gomposite Index. The fitting parabola is

$$
\zeta_{q}=-0.265+q\left(0.6+\lambda^{2}\right)-\lambda^{2} q^{2} / 2 .
$$

where $\lambda^{2} \simeq 0.074$. This indicates that our model has repuduced multifractal scaling properties of price changes.

\section{Conclusion}

Based on the self-organized dynamical evolutionary of the investors structure, a refined dissipation market model is constracted. In BMD model, the existence of the two independent statistical process, one for returns and another for the volatility, may not be very natural. But in this model, the two processes syncretized each other. Furthermore, unlike multifractal cascade-like ideas, this model provides a realistic (agent based) description of financial markets and repuduces multifractal scaling properties of price changes, which indicate that the self-organized dynamical evolutionary of the investors structure may be the origin of the volatility statistical structure.

Acknowledgments. This work has been supported by the National Science Foundation of China under Grant No.70471033 and 70571075, the College Science Foundation of Jiangsu Province (06KJD520122) and the Liu Da Ren Cai Gao Feng Program of Jiangsu Province (06-A-027). 


\section{References}

1. Bachelier L.: Ann. Sci. Ecole Norm. Sup. 3 (1900) 21-86

2. Gopikrishnan P., Plerou V., Amaral L. A. N., et al.: Scaling of the distribution of fluctuations of financial market indices. Phys. Rev. E. 60 (1999) 5305-5316

3. Mantegna R. N., Stanley H. E.: Scaling behaviour in the dynamics of an economic index. Nature. 376 (1995) 46-49

4. Wang B. H., Hui P. M.: The distribution and scaling of fluctuations for Hang Seng index in Hong Kong stock market. Eur. Phys. J. B. 20 (2001) 573-579

5. Ghashghaie S., Breymann W., Peinke J., Talkner P., Dodge Y.: Turbulent Cascades in Foreign Exchange Markets. Nature. 381 (1996) 767-770

6. Mandelbrot B. B.: Fractals and Scaling in Finance. Springer, New York (1997)

7. Schmitt F., Schertzer D., Lovejoy S.: Multifractal analysis of Foreign exchange data. Applied Stochastic Models and Data Analysis. 15 (1999) 29

8. Mandelbrot B. B.: The variation of certain speculative prices. J. Business. 36 (1963) 394

9. Mandelbrot B. B.: Intermittent turbulence in self-similar cascades: divergence of high moments and dimension of the carrier. J. Fluid Mech. 62 (1974) 331

10. Arneodo A., Muzy J. F., Sornette D.: Direct Causal Cascade in the Stock Market. Eur. Phys. J. B. 2 (1998) 277-282

11. Muzy J. F., Delour J., Bacry E.: Modelling fluctuations of financial time series: from cascade process to stochastic volatility model. Eur. Phys. J. B. 17 (2000) 537-548

12. Bacry E., Delour J., Muzy J. F.: Multifractal random walk. Phys. Rev. E. 64 (2001) 026103

13. Cont R., Bouchaud J. P.: Herd behavior and aggregate fluctuations in financial markets. Marcroeconomic dynamics. 4 (2000) 170-196

14. Cavalcante F. S. A., Moreira A. A. et al.: Self-organized percolation growth in regular and disordered lattices. Physica A. 311 (2002) 313-319

15. Stauffer D., Jan N.: Sharp peaks in the percolation model for stock markets. Physica A. 277 (2000) 215-219

16. Kaheman D., Tversky A.: Prospect theory: an analysis of decision under risk. Econometrica. 47 (1979) 263-291

17. Farmer J. D.: Market force, ecology and evolution. Industrial and Corporate Change. 11 (2002) 895-953

18. Muzy J. F., Bacry E., Arneodo A.: The multifractalformalism revisited with wavelets. Int. J. of Bifurcat and Chaos. 4 (1994) 245

19. Borland L., Bouchaud J. P., Muzy J. F., Zumbach G.: The Dynamics of Financial Market_—Mandelbrot's Multifractal Cascades, and Beyond. cond-mat/0501292 v1 